\title{
LA DIFFUSION DE SAVOIRS PAR LA PRESSE SPÉCIALISÉE : MODALITÉS ACTUELLES DES MAGAZINES CONSACRÉS À L'INTERNET
}

\author{
Franck Rebillard ${ }^{1}$
}

Au-delà des instances de formation, spécialisées dans cette activité et qui elles-mêmes peuvent présenter des statuts extrêmement variés (institutions publiques / privées ; formation initiale / continuée ; vocation interne / universelle...), les moyens de transmission du savoir sont à la fois nombreux et diffus. Et au sein de cet ensemble hétérogène des outils pour apprendre, les médias occupent une place relativement importante, sous-estimée par une recherche académique aux découpages disciplinaires bridant souvent l'analyse conjointe de l'éducatif et du communicationnel ${ }^{2}$. Cette carence se ressent avec acuité lorsque l'on tente d'analyser certains types de médias dont la dimension éducative est particulièrement prononcée. Il en est ainsi de la presse spécialisée, et en particulier de celle dédiée aux nouvelles

\footnotetext{
1 Maître de conférences en Sciences de l'information et de la communication à l'Université Lumière Lyon 2.

2 P. Moeglin, "Du mode d'existence des outils pour apprendre", Les enjeux de l'information et de la communication, $\mathrm{n}^{\circ} 1,1999$, http://www.ugrenoble3.fr/les_enjeux/n1/Moeglin/
} 
technologies, qui consacre une large part de sa surface rédactionnelle à des contenus d'ordre didactique.

Les magazines consacrés au thème de l'Internet, "médiations qui mettent en forme ces pratiques"l au même titre que les relations avec l'environnement familial ou professionnel (conseils de l'entourage pour par exemple la transmission intergénérationnelle de compétences au sein du foyer ${ }^{2}$ ), les services d'assistance (les fameuses "hot-lines" qui ne constituent finalement que des télé-services d'après-vente) et bien sûr les organismes de formation, constituent de ce point de vue un terrain d'observation particulièrement intéressant pour aborder la problématique "savoirs formels / informels" :

- ces revues sont très engagées dans la sensibilisation et la formation aux pratiques de l'Internet, au point de souvent diffuser des savoirs informels de façon très formalisée ;

- Internet constituant un objet de savoir relativement récent, au moins pour le "grand public", les savoirs informels distillés par ces revues précèdent les savoirs formels délivrés par les organes de formation institutionnels ;

- ces publications ayant toutes développé des extensions électroniques (sur cédérom ou sur le web), elles proposent de nouvelles formes médiatiques de savoirs informels.

Le développement de ces trois aspects permettra ainsi d'actualiser au travers d'un exemple très récent la place des médias dans la transmission des savoirs, et de s'interroger au final sur les enjeux posés à l'éducation par une telle multiplicité des modalités de sa diffusion.

\section{La presse spécialisée, vecteur médiatique privilégié des savoirs}

De toutes les catégories de journaux ou magazines, la presse spécialisée est sans doute l'une des moins étudiées. Avec la presse gratuite et à un degré moindre la presse locale, elle fait bien souvent figure de parent pauvre de la recherche au regard de la presse nationale d'informations générales. Une telle focalisation peut avoir

1 D. Boullier, C. Charlier, "A chacun son Internet - enquête sur des usages ordinaires", Réseaux, n 86, 1997, p. 165.

2 J. JOUET, D. PASQUIER, "Les jeunes et la culture de l'écran - Enquête nationale auprès des 6-17 ans", Réseaux, n 92-93, 1998, pp. 25-100. 
comme contrepartie un aveuglement scientifique d'autant plus dommageable que la vitalité du secteur de la presse spécialisée ou gratuite ces dernières années n'a d'égale que le fléchissement de la presse d'informations générales.

Au-delà de la disproportion entre l'importance de la presse spécialisée au sein de la presse écrite dans son ensemble et la rareté de son analyse comparée aux nombreux travaux sur les médias, c'est ainsi toute une série de dimensions spécifiques à cette catégorie de publications qui sont passées sous silence ou en tout cas traitées à une injuste hauteur. Et parmi celles-ci, la dimension éducative n'est en général abordée que de façon périphérique au sein d'ouvrages généraux sur la presse écrite, ceux-ci consacrant au mieux une sous-partie à son rôle documentaire ${ }^{1}$ ou à la vulgarisation ${ }^{2}$.

Pourtant, au sein de notre corpus -ensemble des magazines consacrés à l'Internet parus en France entre 1994 et $1998^{3}$-, cette dimension éducative apparaît très nettement. Les revues observées -même si elles peuvent être classées séparément entre la catégorie Presse spécialisée / Grand public / Loisirs / Loisirs informatiques et la catégorie Presse spécialisée / technique et professionnelle / Services marchands / Informatique dans la nomenclature officielle du Service juridique et technique de l'information et de la communication (organisme para-gouvernemental français)- ont en commun une même visée d'encadrement des pratiques de l'Internet.

Certaines pages affichent ainsi très clairement cette orientation didactique, visible dans la reprise du modèle scolaire comme référent :

- Modèle des leçons : certains dossiers sont traités de façon séquentielle, divisés en parties abordées lors des parutions successives de la revue. Est ainsi présente l'idée d'une progression dans l'acquisition des savoirs, et d'une somme de connaissances soumise à un découpage pour être correctement assimilée (dans la revue .net, les différentes "Leçons" sont même subdivisées en "Volets").

1 A. BETEHry (dir.), Revues et magazines d'aujourd'hui. Guide des périodiques à l'intention des bibliothèques publiques, Paris, Éd. du Cercle de la librairie, coll. "Bibliothèques", 1990.

2 J.-M. Charon, La presse en France. De 1945 à nos jours, Paris, Éd. du Seuil, coll. "Points Politique", 1991.

3 Constitué à partir de F. ReBillard, La presse multimédia. Étude de la constitution d'une spécialité médiatique à l'heure de sa diversification sur les nouveaux supports électroniques, Thèse, Université Lumière Lyon 2, juin 1999. 
- Insistance sur la méthode : même si l'action reste la finalité des différents savoirs transmis par l'intermédiaire de ces publications, la réflexion occupe cependant une place prépondérante. Plus précisément se fait jour la nécessité d'une préparation en amont de l'action, devant elle-même s'inscrire dans une démarche rigoureuse illustrée par l'omniprésence de plans d'actions et la numérotation des différentes étapes à suivre (voir en particulier les dossiers consacrés à la création de sites web dans la revue $C D-\operatorname{Rama}$ ).

- Tournure encyclopédique : l'accumulation dans le temps des différents savoirs au sein des magazines se concrétise par la parution d'éditions spéciales ou de numéros hors-série visant à faire le point sur un thème précis. Résultats d'une sédimentation progressive des connaissances des rédacteurs, ces publications s'apparentent ainsi à des ressources documentaires relativement exhaustives, à l'image de Technosphère: "C'est à la fois un magazine permettant de suivre l'actualité technologique et une encyclopédie didactique destinée à expliquer les fonctionnements et l'emploi de ces technologies"1. De façon plus régulière figurent, à la fin de ces magazines, des glossaires et autres lexiques (pour certains très spécifiques, comme par exemple le dictionnaire des "smileys" ou le Langage du Net dans Ariane).

- Omniprésence de l'évaluation : dans le cas des sélections de sites Web en particulier, les magazines distribuent des notes (parfois chiffrées sur 10 points ou sur 20 points, mais le plus souvent sous la forme d'étoiles à la manière des guides gastronomiques ou touristiques comme dans Internet - Le Guide $d u W e b$ ), accompagnées de commentaires d'observation ou d'appréciation. Ces évaluations portent aussi bien sur des sites Web de professionnels que sur ceux d'amateurs, renforçant ainsi l'association à une relation auteur-examinateur entre lecteur et rédacteur de ces revues.

- Adaptation aux différents niveaux : les revues modulent leurs contenus en fonction du degré de pratique, respectant une échelle dont les deux extrémités seraient les débutants et les experts. Cette adaptation est visible par rapport à la typologie d'ensemble des publications (parfois même au sein d'un groupe de presse comme PearsonEdicorp avec ses magazines: Démarrer sur Internet pour les nouveaux venus et .net pour les plus aguerris), et également à l'intérieur de chacune des publications par le biais d'une rubrique "de

1 Extrait de la rubrique "Introduction" du site Technosphère (http://techno.webmaster.fr) en 1997. 
rattrapage" destinée à ceux qui prendraient la formation en cours (ex : "Découvrir" dans Netsurf). Bien entendu, cet effort en direction des néophytes n'est pas exempt de considérations commerciales pour l'éditeur qui par cette stratégie maximise le nombre de lecteurs potentiels.

$\mathrm{Si}$ tous ces éléments montrent de façon assez explicite l'influence du modèle scolaire sur ces magazines, il ne faut pas non plus oublier tous les indices qui signalent que ce modèle constitue un cadre de référence, même par défaut. En effet, très souvent les attributs caricaturaux de ce modèle "classique" se voient transformés ou détournés :

- Le "tableau noir" est remplacé par des supports infographiques ou la reproduction de captures d'écran d'ordinateur. Cependant, les actions d'explicitation sont fondées sur un principe identique même si elles empruntent des formes différentes : à la baguette du "maître" se substitue le pointeur-curseur de la souris, les annotations sont incrustées en couleur, etc.

- La figure du "savant" est également présente même si c'est pour mieux être singée. Ainsi l'emblématique Docteur Web de la revue Planète Internet n'échappe pas aux clichés : représenté sous les traits d'un vieillard à la chevelure ébouriffée et grisonnante, en blouse blanche, toujours confronté à d'étranges créatures sur son écran, ce personnage de bande dessinée semble destiné à dédramatiser le "sérieux" des contenus qui l'entourent dans la page En pratique.

Cette distanciation de la presse magazine vis-à-vis des médiations "standards" du savoir, quitte à jouer avec ses codes, semble se rapprocher des processus de légitimation médiatique du formel remarqués pour d'autres médias'.

1 E. Bevort, Th. De SMEDT, "Le rôle de l'éducation aux médias dans l'appréhension par les jeunes des marques de formalisation médiatique", Colloque "Savoirs formels - Savoirs informels", Université catholique de Louvain, Louvain-la-Neuve, décembre 2000 . 


\section{Internet, objet de savoirs médiatiques et para-médiatiques}

En sortant dans les années 1990 de son cadre militarouniversitaire d'origine, Internet va voir son développement accompagné d'une multitude de discours, selon un mouvement déjà connu pour d'autres innovations socio-techniques apparues ces dernières années dans le domaine de la communication ${ }^{1}: d^{\prime}$ 'abord des discours prospectifs à caractère utopique et / ou révolutionnaire sur la "société de l'information"; ensuite des discours promotionnels présentant les principales applications et les différentes offres matérielles disponibles ; enfin des discours prescriptifs indiquant les utilisations, ou en tout cas celles espérées par les concepteurs.

Même si la presse spécialisée a véhiculé l'ensemble de ces trois types de discours, c'est assurément avec le dernier qu'elle a été au plus près des pratiques et a donc comporté sa plus forte dimension éducative. Plus précisément, elle a dans cette période (1997-98) mis en avant deux pratiques : le surf et la création de sites sur le Web.

Très tôt, les magazines ont décrit le surf sur le Web comme une pratique majeure, voire la principale, d'Internet. Et aussi rapidement, ils en ont accompagné le développement : certes par des contenus didactiques de nature technique (ex : articles exposant les différentes fonctionnalités des logiciels de navigation) ; mais également par une transformation de la revue en guide de navigation, matérialisée par des rubriques ou même des suppléments détachables consistant en des répertoires d'URL (adresses de sites web). Domine alors l'idée que la pérégrination à l'intérieur de la masse d'informations présentes sur l'Internet rend nécessaires des moyens d'orientation, en particulier pour les néophytes. C'est précisément ce que leur apportent des magazines aux noms évocateurs tels que Internet - Le guide $d u W e b$, Ariane, ou Netscope : ils leur servent de boussole à travers une sélection de sites, établissant une véritable cartographie du Web. Les lecteurs-Internautes de ces publications peuvent procéder par ce biais

I J.-G. LACROIX, P. MOEGLIN, "La référence à la convergence des usages : discours promotionnels de Télétel, Vidéoway et la suite", Actes du IXème Congrès national des sciences de l'information et de la communication, Toulouse, S.F.S.I.C., 1994, pp. 297-305. 
à un "digest" des données disponibles, préalable à la consultation du réseau informatique.

La création de sites est l'autre pratique majoritairement prônée par ces revues. Celles-ci s'attachent à donner des méthodes pour la conception des pages web (rubriques de Trucs et astuces liées aux logiciels d'édition), et présentent souvent des modèles de sites à imiter. Une activité dépeinte comme permettant de combiner les sphères du public et du privé, du travail et du temps libre car la création de sites web mêle les aspects professionnels et de loisirs de la pratique de l'Internet, ce hobby pouvant simultanément constituer un métier. Elle amène par exemple les deux revues dominant ce secteur de l'information spécialisée à partir de 1997, Netsurf et .net, à se rejoindre en fusionnant les deux figures de l'entrepreneur et de l'amateur qu'elles mettaient respectivement en avant dans l'espoir de répondre aux attentes de leur lectorat.

Par cette divulgation de savoir-faire autour du surf et de la création web, la presse spécialisée a pu en bien des cas précéder le système scolaire classique. Grâce à son organisation en "entreprisesréseau" décentralisées en "cellules-titres" toujours avides de capter des comportements émergents ${ }^{1}$, la presse magazine possède une capacité de réaction bien supérieure aux institutions de formation qui doivent (notamment pour les institutions publiques de formation initiale et à vocation universelle) respecter un circuit plus long de validation et de consécration des savoirs avant de pouvoir les diffuser.

Cette différence essentielle, responsable d'un tel décalage entre médias et dispositifs institutionnels de formation face aux nouvelles pratiques, se double d'une autre différence quant au statut du savoir. La prise de recul par rapport aux innovations garantit une certaine indépendance du savoir délivré à cette occasion, tandis que la proximité des publications avec l'objet qu'elles décrivent a des conséquences sur la diffusion des savoirs le concernant. Plus précisément, deux cas de figure peuvent se présenter, liés aux activités paramédiatiques des sociétés éditrices de ces revues:

- Le niveau d'objectivité journalistique de ces publications, et donc la neutralité des savoirs transmis par ce biais, va être amoindri par les liens très étroits unissant la plupart des éditeurs avec les

1 J.-M. Charon, La presse magazine, Paris, Éd. La Découverte, coll. "Repères", 1999. 
acteurs économiques du secteur. Et ce d'autant plus dans le secteur de l'Internet, dont beaucoup des plus importants acteurs sont également des groupes de communication présents dans le domaine médiatique. Ainsi, plusieurs des publications étudiées appartiennent de près ou de loin à des fournisseurs d'accès à l'Internet (L'Internet + pour Infonie), des éditeurs de contenus et services en ligne (Internet Accessible, lié à Havas Edition Electronique - Vivendi), ou des fabricants de matériel et logiciels informatiques (Magazine e-business lié à $I B M$ ), adoptant ainsi la forme paroxystique de "consumer magazine". Dans ce cadre, le risque est grand de voir se développer des discours prescriptifs à sens unique, en direction des industriels détenteurs des revues en question.

- L'exploitation commerciale des savoirs acquis par les publications, mais par d'autres moyens que la vente de revues, s'inscrit également dans cette logique de synergie entre médiatisation des savoirs concernant l'Internet et intérêts économiques sur ce marché naissant. Elle est illustrée par la juxtaposition d'organismes de formation et d'étude, qui vont cette fois monnayer directement leur savoir : la cellule de conseil $C D$-Ingéniérie du magazine $C D$-Rama, ou encore le cabinet $I D C$ pour le magazine Webmaster, du groupe $I D G$. L'Internet est également mis à contribution pour cette activité paramédiatique de conseil dans le cas d'Internet Professionnel et de son Observatoire $d u W e b$, qui propose études, analyses des marchés ou encore "tableaux de bord".

\section{De nouveaux supports médiatiques de savoirs}

La presse consacrée à l'Internet a comme spécificité de s'être largement développée sur cédérom et sur le web, entrainant une mise en abîme du contenu et du contenant de ces publications. Quasiment tous les magazines observés disposent d'un complément électronique, pleinement intégré à la ligne éditoriale désormais multi-supports de la publication et donc à sa composante d'encadrement des usages ${ }^{1}$. Les extensions électroniques prolongent le discours de l'édition papier

1 Pour une présentation plus détaillée de ce point, voir F. ReBIllard, "La participation des magazines spécialisés à la formation des usages de l'Internet", Actes du 2ème colloque international "Usages et services des télécommunications", SEE / IREST / ADERA / FT, juin 1999, pp. 127-138. 
dans les faits en fournissant des éléments concrets nécessaires à la pratique de la nouvelle technologie.

Ainsi, les deux principales pratiques de surf et de création web ne bénéficient pas seulement d'un support pédagogique imprimé : leur expérimentation peut également être accompagnée matériellement avec les extensions électroniques des revues spécialisées. Finalement, les versions cédéroms et web des publications consacrées à l'Internet actualisent et dépassent l'expérience de la disquette des prédécesseurs de la presse informatique (qui elle se limitait à la fourniture de logiciels), en se transformant respectivement en :

- Interface de navigation, par la fourniture de logiciels de navigation et de listes sélectives d'URL accessibles hypertextuellement ("bookmarks" ou "favoris"). Pour le surf, les lecteurs-Internautes peuvent se rendre directement aux adresses répertoriées et commentées en profitant des dernières versions des logiciels de navigation disponibles sur les cédéroms vendus avec les magazines et en utilisant les passerelles hypertextuelles figurant dans la présentation cette fois sous forme électronique des différents sites référencés par la publication-guide.

- Plate-forme pour la création, par la fourniture de logiciels d'édition Web et l'exposition de sites Web "modèles". Les logiciels à la pointe de la construction en html ou Java contenus dans les cédéroms sont ainsi chaque mois amenés "sur un plateau" aux lecteurs-Internautes. Les sites web repérés pour leur efficacité comme pour leur beauté ont été téléchargés au préalable, et sont ainsi examinables à loisir par les architectes en herbe ou confirmés. Ceci se réalise en complément aux nombreux conseils d'ordre pédagogique visibles dans la version imprimée, et tend à placer ces magazines en véritables prescripteurs d'usage, tant par les moyens mis à disposition que par les réalisations érigées en modèles.

Avec cet exemple de la presse Internet surviennent de façon plus générale les enjeux liés au développement des technologies d'information et de communication (TIC) pour la diffusion des savoirs. Voir dans quelle mesure les extensions web et cédérom des revues observées s'intègrent aux trois dynamiques - individualisation des pratiques ; dématérialisation de l'offre ; marchandisation du mode de fonctionnement- de la rencontre actuelle entre industries du contenu 
(dont les médias) et TIC ${ }^{1}$, permet notamment de mieux appréhender, en évitant de tomber dans le piège de la futurologie ou du déterminisme technique, les enjeux de cette nouvelle médiation des savoirs :

- L'individualisation des pratiques peut favoriser le recours à l'auto-formation, possible grâce à la lecture des conseils des magazines papier, et renforcée par la mise à disposition des outils, notamment logiciels. Même si l'encadrement humain reste nécessaire comme dans tous les dispositifs d'auto-formation (celle-ci étant toujours assistée quoiqu'à des degrés divers), les individus les plus démunis "cognitivement" par rapport à ce mode d'apprentissage -qui suppose certes une capacité d'autonomie mais également une certaine acculturation à cet encadrement pédagogique minimal (aptitudes à la recherche d'informations; manque de formateurs et d'homologues "apprenants" sur qui se reposer...)-risquent de se retrouver esseulés.

- La dématérialisation de l'offre peut s'illustrer par un déplacement des lieux d'apprentissage. Ceux-ci ne sont plus ni spécifiques -la formation peut avoir lieu hors des enceintes qui généralement abritent cette diffusion des savoirs-, ni exclusifs -l'apprentissage peut prendre place aussi bien au domicile qu'au travail. Mais là encore, les inégalités risquent d'être fortes selon les positions sociales et surtout professionnelles, en fonction de l'équipement matériel, de la disponibilité et une nouvelle fois de l'acculturation à cette formation permanente.

- La marchandisation peut prendre la forme d'une programmation pédagogique par les acteurs du marché. L'exemple des éditeurs de logiciels est symptomatique à cet égard dans la presse consacrée à l'Internet, où la frontière entre rédactionnel et publicitaire est traditionnellement assez mince : les magazines non seulement décrivent en détail les fonctionnalités de leurs produits, mais fournissent également ces mêmes logiciels sur leurs extensions cédérom. Dans ce cas, les visées commerciales influent nécessairement sur la production du savoir, et accentuent en outre une légitimation-certification par le marché, déjà à l'œuvre dans le système scolaire traditionnel même de façon dissimulée ${ }^{2}$.

1 B. MIEGE, Les industries du contenu face à l'ordre informationnel, Grenoble, Presses Universitaires de Grenoble, coll. "La Communication en Plus", 2000.

2 F. Thомas, "Formels, informels : les savoirs "caméléons" ?", Colloque "Savoirs formels - Savoirs informels", Université catholique de Louvain, Louvain-la-Neuve, décembre 2000. 
Au final, l'analyse de ces nouvelles médiations du savoir oblige à concevoir l'interrelation entre les évolutions récentes du domaine de la formation et celles des TIC comme pouvant simultanément et de façon ambivalente :

- activer des dynamiques existantes, les renforcer et les accélérer, ici clairement dans un sens "libéral" avec successivement le renforcement d'une autonomie individualisante, la fusion entre public et privé, et un pilotage par le marché ;

- mais aussi révéler des tendances de fond comme la croissance des inégalités face aux transformations des relations aux savoirs, et rappeler la nécessité d'un service public d'éducation adéquat pour l'ensemble de la population.

Ce qui porte plus globalement à s'interroger sur la nature d'une telle révélation : l'articulation de plus en plus complexe entre savoirs formels et informels et plus généralement le débordement du système éducatif classique tels qu'envisagés dans cet exemple de la presse Internet (savoirs informels présentés de manière formelle ; savoirs formels dépassés par les savoirs informels en certains moments et lieux ; savoirs informels diffusés sur de nouveaux supports, plutôt que les savoirs formels) sont-ils le produit d'une mise en lumière d'évolutions sociales de fond via les TIC ou résultent-ils plutôt d'un manque de recherches académiques préalables sur cette question des savoirs véhiculés par la presse spécialisée ?

Dans ce dernier cas, c'est la mise au rebut d'objets scientifiques jugés illégitimes par l'institution du savoir formel qui constituerait un artefact pour l'étude des savoirs informels. 